\title{
Digitalisierung für das Erreichen der Klimaziele
}

\author{
H. Malleck OVE, C. Mecklenbräuker
}

Online publiziert am 15. Oktober 2020

(c) Springer-Verlag GmbH Austria, ein Teil von Springer Nature 2020

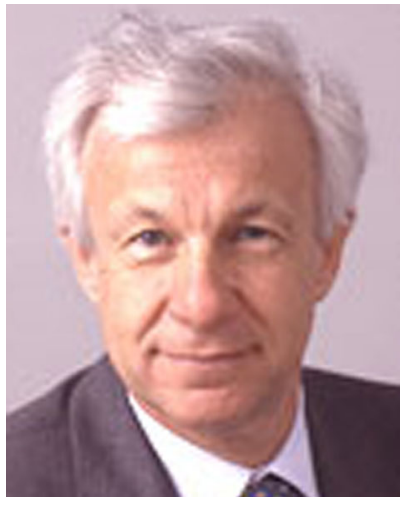

Dr. Helmut Malleck

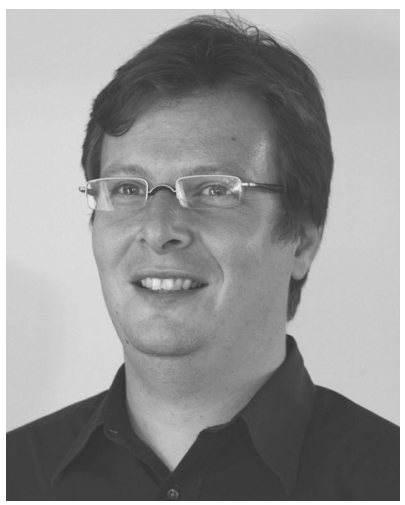

Univ.-Prof. Ing. Dipl.-Ing. Dr.-Ing. Christoph Mecklenbräuker

\begin{abstract}
"Wo der Wille nur erwacht, dort ist schon fast etwas erreicht."
\end{abstract}

Hugo von Hofmannsthal, "Buch der Freunde", 1922

Liebe Leserin und lieber Leser,

bedingt durch die CoronaPandemie ist nichts mehr wie es noch vor einem Jahr war. Wichtige Megatrends - wie Nachhaltigkeit und Klimaschutz - haben kurzfristig bei uns an Relevanz verloren. Zwar konnte eine exponentielle Ausbreitung des Covid-19-Virus in Österreich bisher verhindert werden, dennoch scheint es schwierig, einen Plan für das Erreichen der Klimaziele zu schmieden.

Nur wenn wir den Klimaschutz wirklich wollen, schaffen wir den Wandel.

Im Rückblick: Seit März 2020, nach dem überwundenen, schlagartigen Shutdown, hatte sich zunächst ein Umdenken breitgemacht. Für alles und jedes wurden Alternativen gesucht und gefunden, mit Digitalisierung und Virtualisierung. Mit diesen Optimierungsschritten schien es möglich, eine neue Zukunft zu planen. Lediglich die Pandemie in die Planung miteinzubeziehen, musste misslingen. Unser aller Zukunft ist nunmehr ein Stück weit ungewiss und die Corona-Pandemie wird uns in jeder Hinsicht verändern. Corona wirkt ganz klar als Trendbeschleuniger in Richtung eines neuen Nationalbewusstseins in Europa und verstärkt in Österreich die Regionalisierung. Viele Menschen fürchten um ihre Gesundheit, oftmals auch um ihre wirtschaftliche Existenz. Die ordnungssetzende Macht des Staates ist wieder gefragt und wir werden noch einige Jahre mit den gesundheitlichen, wirtschaftlichen und gesellschaftlichen Folgen des Corona-Virus zurechtkommen müssen.

Vor dem Corona-Shutdown galten die Klimaziele als sinnvoll und erstrebenswert, wenngleich weltweit nicht unumstritten, wäre doch zum Erreichen der Klimaziele eine sofortige, kontrollierte Reduktion weltweiter Treibhausgasemissionen erforderlich. An wissenschaftlichen Erkenntnissen und technologischem Fortschritt mangelt es nicht, um den für das Erreichen der Klimaziele erforderlichen Technologiewechsel voranzutreiben. Dem standen verharmlosende Halbwahrheiten zur Erderwärmung entgegen und auch das Bestreben, etablierte Güter und Dienstleistungen weiterhin gewinnbringend vermarkten zu wollen.

Digitalisierung ist ein wesentlicher Faktor für das Erreichen der Klimaziele. Forschungs- und Entwicklungsergebnisse der Informationsund Kommunikationstechnik lösen einen Innovationsschub aus. Damit werden neue Jobs geschaffen und wirtschaftliche Renditen sind absehbar. Die Digitalisierung bekommt durch die Corona-Krise neue Perspektiven. Einerseits führen unsere Erfahrungen mit Videokonferenzen und im Onlinekonsum zu einer tiefgreifenden Digitalisierung in Wissenschaft, Wirtschaft und Verwaltung sowie in allen privaten Bereichen. Andererseits macht uns die Corona-Krise die Grenzen der Digitalen Revolution (https://de.wikipedia.org/wiki/Digitale_ Revolution, zuletzt zugegriffen 20200906) deutlich: Auch mit für jedermann verfügbarer, nahezu exponentiell ansteigender Information sowie mit weiter erforschter und praktisch eingesetzter Künstlichen Intelligenz, können oftmals präsente Lebensängste nicht überwunden werden.

Das vorliegende Themenheft zeigt das vielfältige Bemühen, dem Klimawandel - trotz Corona-Pandemie - mithilfe der Digitalisierung zu begegnen. Dieses galt es auch beim IT-Kolloquium im Mai 2020 darzustellen. Wegen der Sorge über das unbekannte Corona-Virus wurde das IT-Kolloquium auf 2021 verschoben, die inhaltliche Ausrichtung für das vorliegende Themenheft jedoch beibehalten. Die folgenden Beiträge sind ein ermutigender Zwischenschritt auf dem Weg, den Klimaschutz ernsthaft vorantreiben zu wollen.

\section{Originalarbeiten zum Themenschwerpunkt}

Das vorliegende Themenheft umfasst fünf Originalarbeiten. Um die Brisanz des Klimawandel evident zu halten, wird einleitend ein Faktencheck gegeben. Die folgenden Beiträge spannen den Bogen von Energieerzeugung bis Energieverbrauch im Hinblick auf Digitalisierung und Erreichung der Klimaziele. Ein Beitrag über die klimafreundliche Produktion von Lebensmittel mithilfe der Digitalisierung bildet den Abschluss.

- Dankenswerterweise wurde von Harald Rieder, Universität für Bodenkultur Wien, „Eine Bestandsaufnahme zur Klimakrise inmitten der COVID-19-Pandemie" als Positionspapier für das vorliegende Themenheft verfasst. Das Paper gibt einen fundierten Überblick zu dem aktuell von Menschen herbeigeführten Klimawandel und weist auf langfristige Auswirkungen, selbst nach deutlicher Reduktion von Treibhausgasen, hin. Es ist höchst an der Zeit, noch

Malleck , Helmut, ehemals Arbeitskreis für technische Koordination für öffentliche Kommunikationsnetze und -dienste (AK-TK), Postfach 147, 1006 Wien, Österreich (E-Mail: helmut.malleck@aon.at); Mecklenbräuker, Christoph, Institute of Telecommunications, Technische Universität Wien, Gußhausstraße 25-25a, 1040 Wien, Österreich 
in diesem Jahrzehnt die erforderlichen Maßnahmen zur Emissionsminderung zu setzen.

- Der Beitrag von Friederich Kupzog, et al., AIT Austrian Institute of Technology $\mathrm{GmbH}$, "The role of IT in energy systems: the digital revolution as part of the problem or part of the solution" macht die längst stattfindende Zeitenwende bei Energieversorgungssystemen deutlich, indem real gewordene Möglichkeinen der Digitalisierung mit offenen Systemarchitekturen auf die dezentrale Erzeugung und Verteilung elektrischer Energie abgebildet und kritisch hinterfragt werden.

- Auf Basis einer Open-Source-Modellierungsplattform von Energiedaten macht Hans Auer, et al., TU Wien, "Development and modelling of different decarbonisation scenarios of the European energy system until 2050 as a contribution to achieving the ambitious $1.5^{\circ} \mathrm{C}$ climate target - establishment of open source/data modelling in the European H2020 project openENTRANCE" einige richtungsweisende Aussagen für das Erreichen der europäischen Klimaziele. Dazu werden aus dem openENTRANCE-Projekt vier zukünftig mögliche kohlenstoffarme Übergangspfade der Energieübertragung dargestellt. Nach Vorliegen eines vollständigen offenen Energiesystemmodells wäre auf Basis dieser vier Pfade ableitbar, was im europäischen Energiesystem zukünftig getan werden muss, um einen Beitrag zur Begrenzung der globalen Erwärmung zu leisten.

- Gerhard Christiner, et al., Austrian Power Grid AG, „Das Stromnetz von morgen - Szenarien und Zielbilder für 2040" gibt einen ausgezeichneten Überblick über zukünftige Erfordernisse beim Netzausbau, einerseits durch den verstärkten Einsatz bei der Erzeugung von erneuerbarer Energie und andererseits durch den überregionalen Stromhandel. Digitalisierung unterstützt die dafür erforderliche Flexibilität zur Erreichung der Klimaziele.

- Clemens Gnauer, et al., Forschung Burgenland GmbH, "A recommendation for suitable technologies for an indoor farming framework". Vertikale Landwirtschaft, bei der Pflanzen mithilfe von IKT-Komponenten in vertikaler Anordnung innerhalb von Gebäuden gezüchtet werden, ist eine Möglichkeit, auch trotz des Klimawandels die Menschheit weiterhin mit Lebensmitteln versorgen zu können. Dafür wird der Prototyp einer Testeinheit mit IKT-Infrastruktur und loT-Komponenten vorgestellt, welcher die Wachstumsbedingungen der Pflanzen überwacht und steuert.

\section{Weiterführende Themen in den Berichten}

Aktivitäten zum Klimaschutz bringen Ihnen die folgenden beiden Berichte näher.

- Der Umbau des Energiesystems ist das größte Infrastrukturprojekt des Jahrhunderts und sauberer Strom der Schlüssel zu einer klimafreundlichen Energiezukunft. Vorstandsdirektor Leonhard Schitter, Salzburg AG, macht in seinem Beitrag "Klimaziele nur mit Digitalisierung zu erreichen - Umbau des Energiesystems als größtes Infrastrukturprojekt des Jahrhunderts" mit fünf innovativen Projekten der Energiewirtschaft deutlich, dass die Elektrizitätsversorger neben innovativen Ideen und Digitalisierung auch die passenden Rahmenbedingungen der Politik für die Energiewende benötigen.

- "Silicon Austria Labs forscht an innovativen EBS-Lösungen für die Energiewende": Elektronikbasierte Systeme (EBS) sind Ausgangspunkt für intelligente Produkte und Prozesse für Industrie 4.0, In- ternet of Things (IOT), autonomes Fahren, cyber-physikalische Systeme, Smart City, Smart Energy oder Smart Health. In zahlreichen Projekten widmet sich Silicon Austria Labs GmbH (SAL) dem Klimaschutz. SAL berichtet über das Projekt "Tiny Power Box", bei dem ein bidirektionales Batterieladegerät für E-Autos entwickelt wird, und über das Photovoltaik-Projekt „OptPV4.0” zur Optimierung des Betriebs von Photovoltaikanlagen.

Aus dem Blickwinkel des Ingenieurs haben wir im vorliegenden Themenheft Gegensätzliches in Beziehung zu setzen versucht.

1. Klimawandel: Das Klima auf der Erde war stets im Wandel, hervorgerufen durch Naturgewalten und nun auch vom Menschen. Der Klimawandel, wie er aktuell stattfindet, scheint sich gegen uns Menschen zu richten. Klimabeobachtungen geben Anlass zu großer Sorge. Trotz Corona sollte uns die Umsetzung der als richtig erkannten Maßnahmen zur Erreichung der Klimaziele gelingen. Letztlich ist es aber ein Jahrhundertziel.

2. Digitalisierung lässt mit Künstlicher Intelligenz (KI) eine neue Komplexität entstehen. Mit KI können Entwicklungsprozesse sehr viel schneller vorangetrieben werden. Sind darin einfließende Daten verunreinigt, sind sie vollständig? Werden falsche Ergebnisse erzielt? Jedenfalls haben die Ergebnisse Nachvollziehbarkeitslücken und menschliches Wissen könnte dadurch in Gefahr geraten.

Sowohl die Digitalisierung als auch das Weltklima sind komplex. Komplexe dynamische Systeme könnten im Chaos enden, und es gibt schon jetzt skeptische Stimmen, siehe beispielsweise https:// f3.de/studie-digitalisierung-fuehrt-nicht-zu-mehr-klimaschutz/, zuletzt zugegriffen am 21.09.2020.

Digitalisierung für das Erreichen der Klimaziele? In gewissem Rahmen ist das zweifelsohne ein richtiger Ansatz, vgl. https://www.a1. digital/blog/whitepaper-studie-klimaschutz-und-digitalisierung2019/, zuletzt zugegriffen am 21.09.2020, und erste Schritte wurden bereits gesetzt.

Liebe Leserin und lieber Leser, aktuell ist unter den durch Corona gegebenen Voraussetzungen nur wenig Fortschritt beim Klimaschutz zu erwarten, wenngleich dieser unabdingbar erforderlich wäre. So wird von prominenter Stelle der Wille zum Erreichen der Klimaziele deutlich, etwa in der Rede zur Lage der Union 2020 von Kommissionspräsidentin Ursula von der Leyen, siehe https://ec.europa.eu/info/sites/info/files/soteu_2020_de.pdf, zuletzt zugegriffen am 21.09.2020. Berechtigte Hoffnung für nachfolgende Generationen!

Eine spannende Lektüre der Beiträge zu diesem Themenschwerpunkt wünscht Ihnen

Helmut Malleck und Christoph Mecklenbräuker

\section{Nachschrift von Helmut Malleck:}

Mit diesem, dem 33. von mir zusammengestellten e\&i-Heft, darf ich mich von Ihnen, liebe Leserin und lieber Leser, verabschieden. Ich danke Ihnen für Ihre treue Leserschaft. Es war mir stets ein Anliegen, als Mitglied des Editorial Boards zum "i" unserer Zeitschrift beitragen zu können. Herzlichst, Ihr Helmut Malleck.

Hinweis des Verlags Der Verlag bleibt in Hinblick auf geografische Zuordnungen und Gebietsbezeichnungen in veröffentlichten Karten und Institutsadressen neutral. 